\title{
Prognostic factors for operable biliary tract cancer: serum levels of lactate dehydrogenase, a strong association with survival
}

This article was published in the following Dove Press journal:

OncoTargets and Therapy

Yaodong Zhang, ${ }^{3}$ Tianzhu Qiu,' Biao Wang, 'Wensen Chen, ${ }^{4}$ Xiao Li, ${ }^{5}$ Jing Sun,' Ke Wang, ${ }^{3}$ Xiangcheng $\mathrm{Li}^{3}{ }^{3}$ Yanhong Gu,' Yongqian Shu, ${ }^{1,6}$ Xiaofeng Chen'

'Department of Oncology, The First Affiliated Hospital of Nanjing Medical University, Nanjing, People's Republic of China; ${ }^{2}$ Department of Biological Therapy, Eastern Hepatobiliary Surgery Hospital Affiliated to Second Military Medical University, Shanghai, People's Republic of China; ${ }^{3}$ Liver Transplantation Center, The First Affiliated Hospital of Nanjing Medical University, Nanjing, People's Republic of China; ${ }^{4}$ Department of Infection Management Office, The First Affiliated Hospital of Nanjing Medical University, Nanjing, People's Republic of China; ${ }^{5}$ Department of Pathology, The First Affiliated Hospital of Nanjing Medical University, Nanjing, People's Republic of China; ' Jiangsu Key Lab of Cancer Biomarkers, Prevention and Treatment, Collaborative Innovation Center for Cancer Personalized Medicine, Nanjing Medical University, Nanjing, People's Republic of China

*These authors contributed equally to this work
Ling Ma, I,* Jinrong Qiu, ${ }^{2, *}$

Background: Biliary tract cancers (BTCs) are uncommon but fatal, with a low 5-year survival rate after surgical resection. This study was designed to investigate the prognostic factors for operable BTC.

Methods: Baseline demographics at diagnosis were retrospectively evaluated in 341 BTC patients undergoing radical surgery at The First Affiliated Hospital of Nanjing Medical University from January 2011 to December 2015. The association between prognostic factors and overall survival (OS) was determined by multivariate analysis using the Cox proportional hazards regression model.

Results: Our study showed that 341 patients were included in the analysis, of which 166 (48.7\%) were males and $175(51.3 \%)$ were females. Older age, depth of tumor invasion, positive surgical margin, lower hemoglobin, and higher lactic dehydrogenase (LDH) were associated with significantly worse OS using multivariate analysis. In the entire cohort, the estimate of median OS in patients with LDH $<271 \mathrm{U} / \mathrm{L}$ was 36.291 months $(95 \%$ CI; 30.989-41.594 months), and 30.736 months (95\% CI; 19.154-42.318 months) in patients with $\mathrm{LDH} \geq 271 \mathrm{U} / \mathrm{L}$ (adjusted HR-1.505, 95\% CI; 1.009-2.245, $P=0.045$ ). Moreover, it was investigated whether serum LDH retained its significance as a prognostic marker in BTC subgroups separately. The results showed that LDH was prognostic in patients with distal bile duct (DBD) carcinoma undergoing radical surgery (HR-2.452, 95\% CI; 1.167-5.152, $P=0.018$ ). However, there were no statistical differences between LDH and OS in multivariate analysis in the other three individual subgroups except for DBD carcinoma. This may be due to the limited number of patients in the study, indicating that a greater number of patients may be required for statistical significance.

Conclusion: Older age, depth of tumor invasion, positive surgical margin status, lower hemoglobin levels, and elevated serum LDH level are associated with poor survival in operable BTC patients. Serum LDH level is a cost-effective prognostic biomarker in patients with operable BTC and especially DBD carcinoma.

Keywords: biliary tract cancer, lactate dehydrogenase, tumor marker, prognosis, radical surgery

\section{Introduction}

Correspondence: Xiaofeng Chen; Yongqian Shu Department of Oncology, The First Affiliated Hospital of Nanjing Medical University, 300 Guangzhou Road, Nanjing 210029, People's Republic of China Tel +86 I35 $85172066 ;+862568136428$ Fax +86 25 837I 0040; +86 2568136428 Email xiaofengch198019@126.com; shuyongqian@csco.org.cn
Biliary tract cancers (BTCs) are rare but fatal. In the USA in 2017, the estimated number of new cancer cases from gallbladder and other biliary tissue was 11,740 (5,320 in males; 6,420 in females) and the estimated number of deaths was 3,830 (1,630 in males; 2,200 in females). ${ }^{1}$ In People's Republic of China in 2015, estimates of gallbladder cancer incidence and mortality were 52,800 (24,500 in males; 
28,300 in females) and 40,700 (18,800 in males; 21,800 in females), respectively. ${ }^{2}$ Tumors of the biliary tract typically have a poor prognosis, with 5-year survival rates in the range of 5\%-15\%. ${ }^{3,4}$ According to the primary site, BTCs encompass gallbladder carcinoma (GBC), distal bile duct (DBD) carcinoma, intrahepatic cholangiocarcinoma (IHC), and hilar cholangiocarcinoma.

Surgical resection is reported to be the only potential method for curative treatment of BTC. ${ }^{5,6}$ Data supporting an adjuvant approach are sparse. The Phase III UK Advanced Biliary Cancer-02 (ABC-02) study confirmed the combination of cisplatin with gemcitabine as the standard treatment for advanced BTC. ${ }^{7}$ Targeting angiogenesis and HER2/ neu blockade have been shown to be promising treatment strategies for BTC patients. ${ }^{8,9}$ However, prognostic factors among BTC patients remain scarce. R0 resection and adjuvant chemotherapy may be prognosticators of long-term survival for patients with cholangiocarcinoma. ${ }^{10-12}$ Despite surgical resection, recent reports concerning postoperative prognosis are unsatisfactory, with a 5-year survival rate of $27 \%-37 \%$ for DBD carcinoma, $22 \%-44 \%$ for IHC, and $11 \%-41 \%$ for hilar cholangiocarcinoma. ${ }^{13-17}$ Therefore, the prognostic factors that predict long-term survival of BTC patients undergoing radical surgery are essential.

Serum tumor markers, including carbohydrate antigen 19-9 (CA19-9) and carcinoembryonic antigen (CEA), have been used as a complementary approach for the diagnosis of BTC in conjunction with radiology and histology/ cytology. ${ }^{18-20}$ Likewise, lactic dehydrogenase (LDH) is a glycolytic enzyme that is essential for tumor maintenance and can be used as an attractive antitumor strategy by inhibiting glucose metabolism. ${ }^{21} \mathrm{LDH}$ is reported to be associated with the hypoxia-inducible factor (HIF) pathway, hypoxia, acidity, and neoangiogenesis. ${ }^{22}$ Serum LDH levels can provide prognostic information on overall survival (OS) in patients with advanced pancreatic cancer, colorectal cancer, and melanoma. ${ }^{23-26}$ However, the prognostic roles of tumor markers including CEA, CA19-9, and LDH have not yet been clearly elucidated in operable BTC.

The aim of this study was to investigate the role of tumor markers in predicting clinical outcome for BTC patients who have undergone radical surgery. It was hypothesized that elevated baseline serum LDH is prognostic of diminished OS in operable BTC, especially in operable DBD carcinoma.

\section{Methods}

\section{Study population and data collection}

Patients who were diagnosed with BTC at The First Affiliated Hospital of Nanjing Medical University from
January 2011 to December 2015 were enrolled in this study. All patients were histologically confirmed with BTC. Due to a potentially different biology, ${ }^{27}$ ampullary tumors were not included in this study. Exclusion criteria were as follows: 1) BTC patients with concurrent primary tumors of other types, 2) patients with missing or incomplete interest data, 3) patients with advanced BTC, and 4) patients who expired within the first month after surgery due to postoperative complications. In total, 341 patients were eligible for the analysis and were followed until December 2016 (Figure 1).

The baseline demographics that were evaluated in this study included age, sex, site of primary tumor (DBD, GBC, IHC, or hilar), depth of tumor invasion, lymph node status, metastasis, stage, histologic differentiation, lymphovascular invasion, perineural invasion, surgical margin status, hemoglobin, CEA, CA19-9, and LDH at diagnosis (prior to surgery). A CEA level of $4.7 \mathrm{ng} / \mathrm{mL}$, CA 19-9 level of

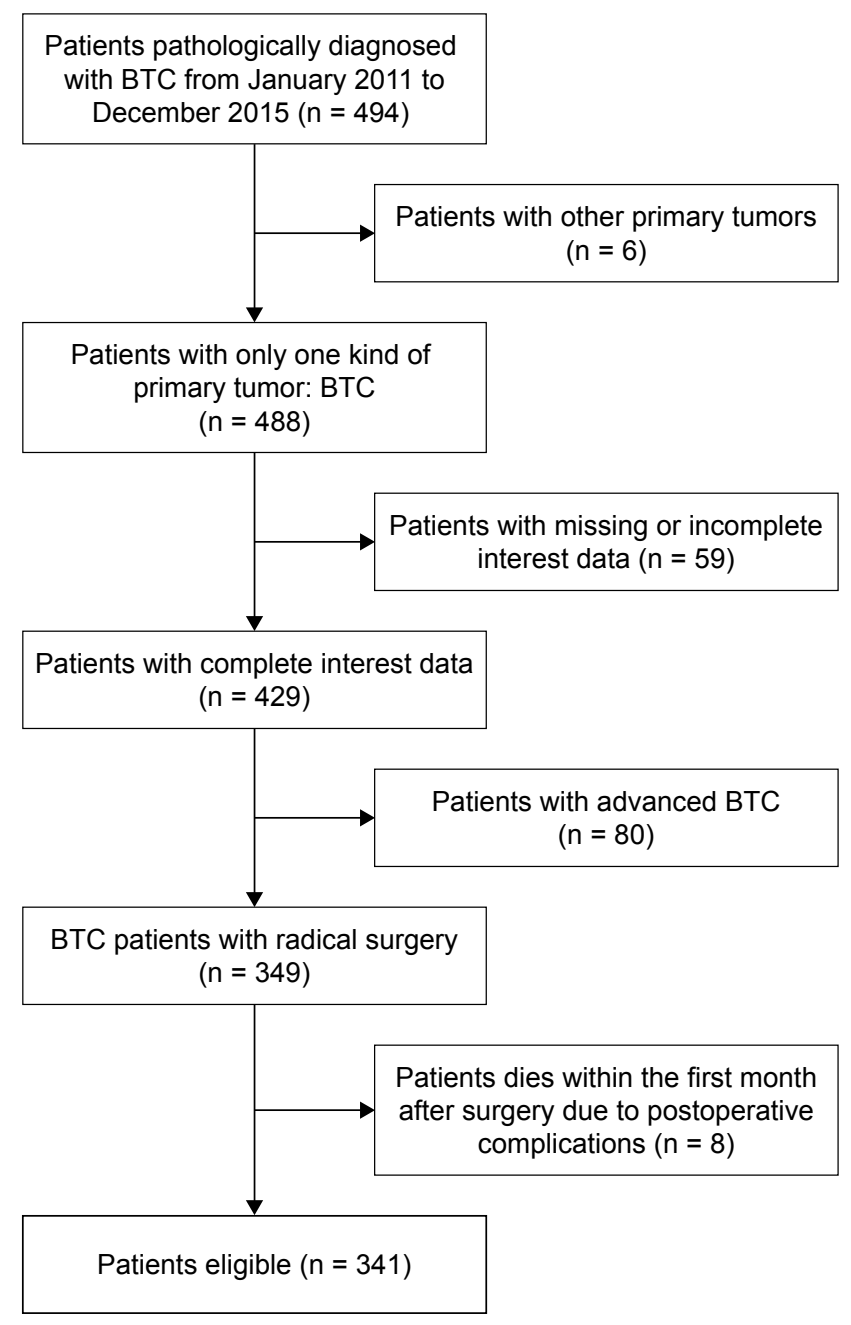

Figure I Flowchart on patient selection. Abbreviation: BTC, biliary tract cancer. 
$39 \mathrm{U} / \mathrm{mL}$, and LDH level of $271 \mathrm{U} / \mathrm{L}$ were defined as cutoff values for normal levels according to the historical data and manufacturer's recommendations. ${ }^{28,29}$ Stage classification was based on pathological findings and documented according to the seventh edition of the Union for International Cancer Control tumor-node-metastasis staging system. ${ }^{30}$ The protocol was approved by the Ethics Committee of The First Affiliated Hospital of Nanjing Medical University prior to study initiation and conformed to the provisions of the Declaration of Helsinki (as revised in Fortaleza, Brazil, October 2013). Written informed consent was obtained from all patients.

\section{Statistics}

Continuous variables were described as median (interquartile range [IQR]). OS was defined as the period from the day of operation to death or the last follow-up (December 2016). The OS of all patients in this study was analyzed and recorded. Survival distribution was estimated by the Kaplan-Meier method. The log-rank test was used to evaluate the equality of survivor functions across groups. The Cox proportional hazards model was used with a 95\% CI for the univariate and multivariate analyses. The chi-square test was used to compare baseline characteristics of patients between the LDH groups. All statistical tests were two-sided. Statistical significance was defined as $P<0.05$. All analyses were performed using SPSS software for Windows (version 19; IBM SPSS, Somers, NY, USA).

\section{Results}

\section{Patients}

A total of 341 BTC patients were available for our analysis. Patient and tumor characteristics of the entire cohort are presented in Table 1. Among the 341 patients, 146 (42.8\%) patients were above 65 years of age and 195 (57.2\%) patients were below 65 years of age. The study cohort consisted of $166(48.7 \%)$ males and 175 (51.3\%) females. The median serum levels of CEA, CA19-9, and LDH of the entire cohort were $3.40 \mathrm{ng} / \mathrm{mL}$ (IQR 2.11-5.27 ng/mL), 83.2 U/mL (IQR 24.8-417.2 U/mL), and $215 \mathrm{U} / \mathrm{L}$ (IQR 184-273 U/L), respectively.

Patient characteristics by primary site are reported in Table 2. Malignancies included 98 (28.7\%) DBD carcinoma, 127 (37.2\%) GBC, 38 (11.1\%) IHC, and 75 (22.0\%) hilar cholangiocarcinoma.

\section{Outcomes in the entire cohort}

To investigate whether the patient baseline characteristics and laboratory factors were associated with survival of
Table I Demographics for entire cohort of patients with biliary tract cancer $(\mathrm{N}=34 \mathrm{I})$

\begin{tabular}{|c|c|}
\hline Variable & $\begin{array}{l}\text { Entire cohort } \\
(\mathbf{N}=34 \mathrm{I}) \\
\mathbf{N}(\%)\end{array}$ \\
\hline \multicolumn{2}{|l|}{ Age } \\
\hline$<65$ years & $195(57.2)$ \\
\hline$\geq 65$ years & $146(42.8)$ \\
\hline \multicolumn{2}{|l|}{ Sex } \\
\hline Male & $166(48.7)$ \\
\hline Female & $175(51.3)$ \\
\hline \multicolumn{2}{|l|}{ Primary tumor site } \\
\hline Distal bile duct & $98(28.7)$ \\
\hline Gallbladder & $127(37.2)$ \\
\hline Intrahepatic & $38(I I . I)$ \\
\hline Hilar & $75(22.0)$ \\
\hline NA & $3(0.9)$ \\
\hline \multicolumn{2}{|l|}{ Depth of tumor invasion } \\
\hline $\mathrm{TI}$ & $23(6.7)$ \\
\hline $\mathrm{T} 2$ & $132(38.7)$ \\
\hline T3 & $158(46.3)$ \\
\hline $\mathrm{T} 4$ & $15(4.4)$ \\
\hline NA & $13(3.8)$ \\
\hline \multicolumn{2}{|l|}{ Lymph node status } \\
\hline Negative & $223(65.4)$ \\
\hline Positive & $109(32.0)$ \\
\hline NA & $9(2.6)$ \\
\hline \multicolumn{2}{|l|}{ Metastasis } \\
\hline Negative & $299(87.7)$ \\
\hline Positive & $33(9.7)$ \\
\hline NA & $9(2.6)$ \\
\hline \multicolumn{2}{|l|}{ Seventh UICC TNM Stage } \\
\hline I & $4 I(12.0)$ \\
\hline ॥ & $138(40.5)$ \\
\hline III & $100(29.3)$ \\
\hline IV & $46(13.5)$ \\
\hline NA & $16(4.7)$ \\
\hline \multicolumn{2}{|l|}{ Histologic differentiation } \\
\hline Well and moderately differentiated & I5I (44.2) \\
\hline Poorly differentiated & $169(49.6)$ \\
\hline NA & $21(6.2)$ \\
\hline \multicolumn{2}{|l|}{ Lymphovascular invasion } \\
\hline Negative & $296(86.8)$ \\
\hline Positive & $39(11.4)$ \\
\hline NA & $6(1.8)$ \\
\hline \multicolumn{2}{|l|}{ Perineural invasion } \\
\hline Negative & $172(50.4)$ \\
\hline Positive & $163(47.8)$ \\
\hline NA & $6(1.8)$ \\
\hline \multicolumn{2}{|l|}{ Resection margin } \\
\hline Negative & $258(75.7)$ \\
\hline Positive & $77(22.6)$ \\
\hline NA & $6(1.8)$ \\
\hline Hemoglobin g/L, median (IQR) & $125(114-135)$ \\
\hline CEA ng/mL, median (IQR) & $3.40(2.11-5.27)$ \\
\hline CAI9-9 U/mL, median (IQR) & $83.2(24.8-4 \mid 7.2)$ \\
\hline LDH U/L, median (IQR) & $215(184-273)$ \\
\hline
\end{tabular}

Abbreviations: NA, not available; UICC, Union for International Cancer Control; TNM, tumor node metastasis; IQR, interquartile range; CEA, carcinoembryonic antigen; CAI9-9, carbohydrate antigen 19-9; LDH, lactic dehydrogenase. 
Table 2 Demographics by primary site for entire cohort of patients with biliary tract cancer $(\mathrm{N}=338)$

\begin{tabular}{|c|c|c|c|c|}
\hline Variable & $\begin{array}{l}\text { Distal bile duct } \\
(\mathbf{N}=98) \\
\mathbf{N}(\%)\end{array}$ & $\begin{array}{l}\text { Gallbladder } \\
(\mathbf{N}=127) \\
N(\%)\end{array}$ & $\begin{array}{l}\text { Intrahepatic } \\
(\mathbf{N}=38) \\
\mathbf{N}(\%)\end{array}$ & $\begin{array}{l}\text { Hilar } \\
(\mathbf{N}=75) \\
\mathbf{N}(\%)\end{array}$ \\
\hline \multicolumn{5}{|l|}{ Age } \\
\hline$<65$ years & $57(58.2)$ & $68(53.5)$ & $25(65.8)$ & $45(60.0)$ \\
\hline$\geq 65$ years & $4 \mid(4 I .8)$ & $59(46.5)$ & $13(34.2)$ & $30(40.0)$ \\
\hline \multicolumn{5}{|l|}{ Sex } \\
\hline Male & $52(53.1)$ & $47(37.0)$ & $19(50)$ & $47(62.7)$ \\
\hline Female & $46(46.9)$ & $80(63.0)$ & $19(50)$ & $28(37.3)$ \\
\hline \multicolumn{5}{|l|}{ Depth of tumor invasion } \\
\hline TI & $2(2.0)$ & $7(5.5)$ & $12(31.6)$ & $2(2.7)$ \\
\hline $\mathrm{T} 2$ & $29(29.6)$ & $48(37.8)$ & $13(34.2)$ & $42(56.0)$ \\
\hline T3 & $62(63.3)$ & $64(50.4)$ & $9(23.7)$ & $23(30.7)$ \\
\hline $\mathrm{T} 4$ & $2(2.0)$ & $6(4.7)$ & I (2.6) & $6(8.0)$ \\
\hline NA & $3(3.1)$ & $2(1.6)$ & $3(7.9)$ & $2(2.7)$ \\
\hline \multicolumn{5}{|l|}{ Lymph node status } \\
\hline Negative & $66(67.3)$ & $78(6 I .4)$ & $28(73.7)$ & $51(68.0)$ \\
\hline Positive & $31(31.6)$ & $48(37.8)$ & $7(18.4)$ & $22(29.3)$ \\
\hline NA & $\mathrm{I}(\mathrm{I} .0)$ & $\mathrm{I}(0.8)$ & $3(7.9)$ & $2(2.7)$ \\
\hline \multicolumn{5}{|l|}{ Metastasis } \\
\hline Negative & $93(94.9)$ & $110(86.6)$ & $28(73.7)$ & $67(89.3)$ \\
\hline Positive & $4(4.1)$ & $16(12.6)$ & 7 (I8.4) & $6(8.0)$ \\
\hline NA & I (I.0) & I (0.8) & $3(7.9)$ & $2(2.7)$ \\
\hline \multicolumn{5}{|l|}{ Seventh UICC TNM stage } \\
\hline I & $20(20.4)$ & II (8.7) & $8(21.1)$ & $2(2.7)$ \\
\hline II & $58(59.2)$ & $37(29.1)$ & $10(26.3)$ & $33(44.0)$ \\
\hline III & $13(13.3)$ & $56(44.1)$ & $4(10.5)$ & $27(36.0)$ \\
\hline IV & $4(4.1)$ & $20(15.7)$ & II (28.9) & II (I4.7) \\
\hline NA & $3(3.1)$ & $3(2.4)$ & $5(13.2)$ & $2(2.7)$ \\
\hline \multicolumn{5}{|l|}{ Histologic differentiation } \\
\hline Well and moderately differentiated & $49(50.0)$ & $56(44.1)$ & $16(42.1)$ & $30(40.0)$ \\
\hline Poorly differentiated & $43(43.9)$ & $65(5 \mathrm{I} .2)$ & $20(52.6)$ & $4 \mathrm{I}(54.7)$ \\
\hline NA & $6(6.1)$ & $6(4.7)$ & $2(5.3)$ & $4(5.3)$ \\
\hline \multicolumn{5}{|l|}{ Lymphovascular invasion } \\
\hline Negative & $88(89.8)$ & $109(85.8)$ & $35(92.1)$ & $64(85.3)$ \\
\hline Positive & $10(10.2)$ & $17(13.4)$ & $2(5.3)$ & $10(13.3)$ \\
\hline NA & 0 & I (0.8) & I (2.6) & $\mathrm{I}(\mathrm{I} .3)$ \\
\hline \multicolumn{5}{|l|}{ Perineural invasion } \\
\hline Negative & $45(45.9)$ & $84(66.1)$ & $22(57.9)$ & $21(28.0)$ \\
\hline Positive & $53(54.1)$ & $42(33.1)$ & $15(39.5)$ & $53(70.7)$ \\
\hline NA & 0 & $\mathrm{I}(0.8)$ & $I(2.6)$ & $\mathrm{I}(\mathrm{I} .3)$ \\
\hline \multicolumn{5}{|l|}{ Resection margin } \\
\hline Negative & $76(77.6)$ & $100(78.7)$ & $31(81.6)$ & $51(68.0)$ \\
\hline Positive & $22(22.4)$ & $26(20.5)$ & $6(15.8)$ & $23(30.7)$ \\
\hline NA & 0 & I (0.8) & I (2.6) & $\mathrm{I}(\mathrm{l} .3)$ \\
\hline Hemoglobin g/L, median (IQR) & $124.50(|1| .00-134.25)$ & $125.00(113.00-134.00)$ & $127(117.75-139.25)$ & $124.00(113.00-135.00)$ \\
\hline CEA ng/mL, median (IQR) & $3.65(2.32-4.93)$ & $3.42(1.90-6.93)$ & $3.30(2.00-6.44)$ & $3.10(2.13-5.15)$ \\
\hline CAI9-9 U/mL, median (IQR) & $130.90(49.20-4 \mid 8.90)$ & $40.01(11.93-279.75)$ & $69.60(24.90-1,000)$ & $204.00(48.60-606.40)$ \\
\hline LDH U/L, median (IQR) & $219.50(183.00-300.25)$ & $204.00(177.00-250.00)$ & 222.50 (I82.00-273.00) & 222.00 (194.00-267.0) \\
\hline
\end{tabular}

Abbreviations: NA, not available; UICC, Union for International Cancer Control; TNM, tumor node metastasis; IQR, interquartile range; CEA, carcinoembryonic antigen; CA 19-9, carbohydrate antigen 19-9; LDH, lactic dehydrogenase.

BTC patients undergoing radical surgery, univariate and multivariate analyses were performed. Univariate analysis revealed that better survival was associated with age $<65$ years, with a tumor primary site from DBD, depth of tumor invasion, negative lymph node status, early stage, well and moderately differentiated histological differentiation, absence of lymphovascular invasion, absence of perineural invasion, negative surgical margin, hemoglobin $\geq 110 \mathrm{~g} / \mathrm{L}$, and LDH $<271 \mathrm{U} / \mathrm{L}$ 
Table 3 Univariate and multivariate analyses of patients with biliary tract cancer $(\mathrm{N}=34 \mathrm{I})$

\begin{tabular}{|c|c|c|c|c|c|c|}
\hline \multirow[t]{2}{*}{ Variable } & \multicolumn{3}{|c|}{ Univariate analysis } & \multicolumn{3}{|c|}{ Multivariate analysis } \\
\hline & HR & $95 \% \mathrm{Cl}$ & $P$-value & HR & $95 \% \mathrm{Cl}$ & $P$-value \\
\hline Age $\geq 65$ years vs $<65$ years & 1.457 & $1.087-1.953$ & 0.012 & 1.491 & $1.060-2.099$ & 0.022 \\
\hline Female vs male & $\mathrm{I} .247$ & $0.930-1.672$ & 0.140 & 1.094 & $0.764-1.565$ & 0.624 \\
\hline GBC vs DBD & $\mathrm{I} .547$ & $1.066-2.246$ & 0.022 & 1.440 & $0.928-2.236$ & 0.104 \\
\hline $\mathrm{IHC}$ vs DBD & 1.321 & $0.778-2.242$ & 0.303 & 1.826 & $0.905-3.686$ & 0.093 \\
\hline Hilar vs DBD & 1.470 & $0.957-2.257$ & 0.078 & 1.326 & $0.791-2.223$ & 0.284 \\
\hline \multicolumn{7}{|l|}{ Depth of tumor invasion } \\
\hline T2 vs TI & 2.565 & $1.032-6.377$ & 0.043 & 2.730 & $0.931-8.001$ & 0.067 \\
\hline T3 vs TI & 3.376 & I.372-8.304 & 0.008 & 3.343 & I.138-9.818 & 0.028 \\
\hline T4 vs TI & 5.827 & $2.020-16.806$ & 0.001 & 5.381 & $1.565-18.505$ & 0.008 \\
\hline Lymph node status (positive vs negative) & 1.727 & $1.275-2.338$ & $<\mathbf{0 . 0 0 1}$ & 1.357 & $0.948-1.944$ & 0.096 \\
\hline Metastasis (positive vs negative) & 1.383 & $0.867-2.205$ & 0.173 & 1.018 & $0.587-1.767$ & 0.949 \\
\hline Stage II vs I & 2.599 & $1.339-5.044$ & 0.005 & I & I & I \\
\hline Stage III vs I & 3.627 & I.862-7.067 & $<0.001$ & 1 & I & I \\
\hline Stage IV vs I & 4.218 & $2.060-8.637$ & $<0.001$ & 1 & I & I \\
\hline $\begin{array}{l}\text { Histologic differentiation (poorly vs well and } \\
\text { moderately differentiated) }\end{array}$ & 1.745 & $1.287-2.366$ & $<0.001$ & 1.409 & $0.991-2.005$ & 0.056 \\
\hline Lymphovascular invasion (positive vs negative) & $\mathrm{I} .74 \mathrm{I}$ & $1.155-2.626$ & 0.008 & 1.211 & $0.739-1.984$ & 0.447 \\
\hline Perineural invasion (positive vs negative) & 1.387 & $1.03 \mid-1.867$ & 0.031 & 0.973 & $0.673-1.407$ & 0.885 \\
\hline Resection margin (positive vs negative) & 1.557 & $1.108-2.187$ & 0.011 & 1.500 & $1.01 \mathrm{I}-2.227$ & 0.044 \\
\hline Hemoglobin $(<110 \mathrm{~g} / \mathrm{L}$ vs $\geq 110 \mathrm{~g} / \mathrm{L})$ & 1.805 & $1.280-2.546$ & 0.001 & $1.55 \mid$ & $1.010-2.382$ & 0.045 \\
\hline CEA $(\geq 4.7 \mathrm{ng} / \mathrm{mL}$ vs $<4.7 \mathrm{ng} / \mathrm{mL})$ & 1.216 & $0.875-1.69 \mid$ & 0.244 & 1.442 & $0.999-2.082$ & 0.051 \\
\hline CAI9-9 ( $\geq 39 \mathrm{U} / \mathrm{mL}$ vs $<39 \mathrm{U} / \mathrm{mL})$ & 1.340 & $0.945-1.899$ & 0.101 & 0.972 & $0.646-1.463$ & 0.893 \\
\hline $\mathrm{LDH}(\geq 27 \mid \mathrm{U} / \mathrm{L}$ vs $<27$ I U/L) & 1.435 & $1.038-1.982$ & 0.029 & 1.505 & $1.009-2.245$ & 0.045 \\
\hline
\end{tabular}

Notes: Data in bold indicates $P<0.05$. / indicates not included in the multivariate analysis.

Abbreviations: GBC, gallbladder carcinoma; DBD, distal bile duct; IHC, intrahepatic carcinoma; CEA, carcinoembryonic antigen; CAI9-9, carbohydrate antigen I9-9; $\mathrm{LDH}$, lactic dehydrogenase.

(Table 3 and Figure 2). However, using univariate analysis, sex, metastasis, CEA, and CA19-9 were not statistically significant in OS in patients with radical surgery of BTC. Compared to $\mathrm{LDH}<271 \mathrm{U} / \mathrm{L}, \mathrm{LDH} \geq 271 \mathrm{U} / \mathrm{L}$ was associated with a worse OS using univariate analysis (HR-1.435, 95\% CI; 1.038-1.982, $P=0.029$ ). Moreover, LDH retained its significance as a prognostic marker in multivariate analysis (HR-1.505, 95\% CI; 1.009-2.245, $P=0.045$ ) in the entire cohort along with age, depth of tumor invasion, resection margin, and hemoglobin (Table 3).

\section{Comparison between high and low LDH groups}

For all patients included in the analysis, the estimate of median OS in patients with LDH <271 U/L was 36.291 months (95\% CI; 30.989-41.594 months) and 30.736 months (95\% CI; 19.154-42.318 months) in patients with $\mathrm{LDH} \geq 271 \mathrm{U} / \mathrm{L}$ (log-rank $P=0.028$ ) (Figure $2 \mathrm{E})$.

Patients showing a pretreatment serum LDH $<271 \mathrm{U} / \mathrm{L}$ were classified as LDH-low patients (251 patients, 73.6\%, group A), whereas patients with pretreatment serum LDH level $\geq 271 \mathrm{U} / \mathrm{L}$ were classified as LDH-high patients
(90 patients, 26.4\%, group B). The patients with positive lymphovascular invasion and positive perineural invasion were more frequently found in the high LDH group $(P=0.010$, $P=0.031$, respectively) (Table 4).

\section{Outcomes by BTC site}

Considering the tumor primary site, it was further determined whether serum LDH retained its significance as a prognostic marker in BTC subgroups separately. The results showed that $\mathrm{LDH}$ was prognostic in multivariate analysis for the DBD subgroup (HR-2.452, 95\% CI; 1.167-5.152, $P=0.018$ ) (Table 5). The data showed a nonsignificant improvement in survival with $\mathrm{LDH}<271 \mathrm{U} / \mathrm{L}$ compared with $\mathrm{LDH} \geq 271 \mathrm{U} / \mathrm{L}$ in multivariate model in individual subgroups: GBC (Table S1) or hilar cancer (Table S2). Moreover, in multivariate analysis, age $(P=0.003)$ and depth of tumor invasion $(P=0.020)$ showed a significant association with OS in GBC (Table S1). Since there was a limited number of patients in the IHC subgroup, the corresponding multivariate analysis was not available. Details of multivariate analysis outcomes for OS by BTC site are provided in Tables 5, S1, and S2. 
A

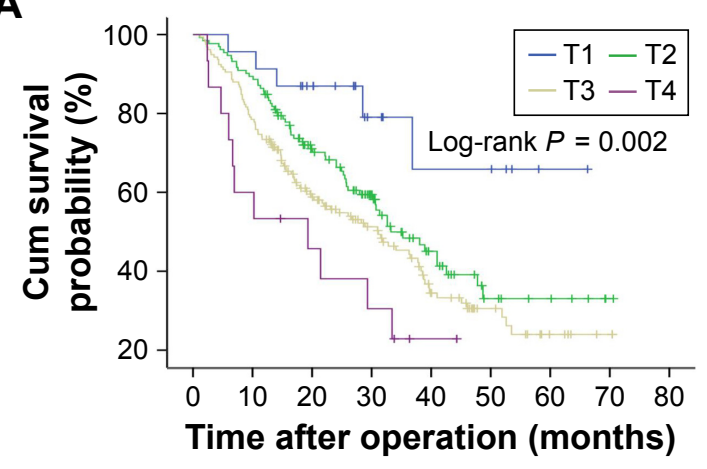

Number at risk

$\begin{array}{lllllllll}\text { T1 } & 23 & 22 & 17 & 8 & 5 & 5 & 1 & \\ \text { T2 } & 132 & 118 & 76 & 49 & 24 & 9 & 6 & 1 \\ \text { T3 } & 158 & 124 & 78 & 54 & 29 & 15 & 6 & 2 \\ \text { T4 } & 15 & 9 & 6 & 4 & 1 & & & \end{array}$

C

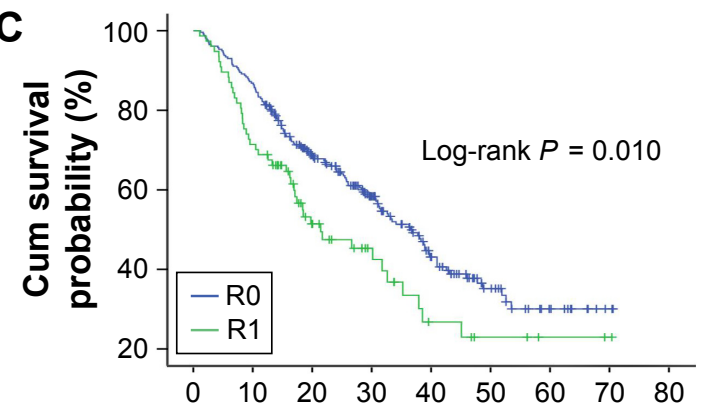

Time after operation (months)

Number at risk

$\begin{array}{lllllllll}\text { R0 } & 258 & 224 & 152 & 100 & 53 & 25 & 11 & 2\end{array}$

$\begin{array}{lllllllll}\mathrm{R} 1 & 77 & 55 & 28 & 16 & 7 & 4 & 2 & 1\end{array}$
B

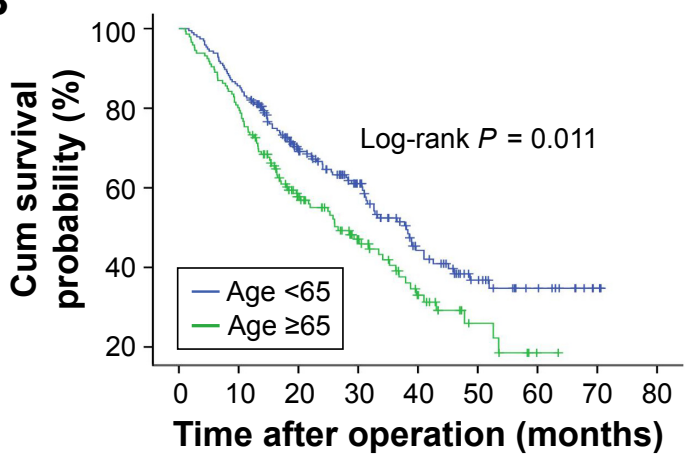

Number at risk

$\begin{array}{lllllllll}\text { Age }<65 & 195 & 167 & 114 & 75 & 40 & 22 & 12 & 3\end{array}$

$\begin{array}{llllllll}\text { Age } \geq 65 & 146 & 117 & 67 & 41 & 20 & 7 & 1\end{array}$

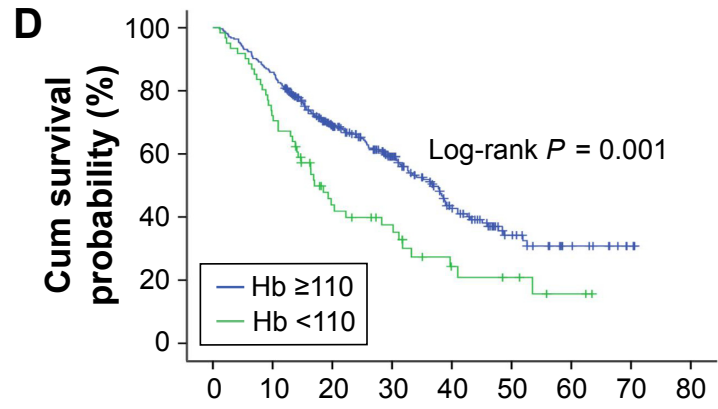

Time after operation (months)

Number at risk

$\begin{array}{lllllllll}\text { Pre-Hb } \geq 110 & 251 & 237 & 156 & 98 & 51 & 23 & 11 & 3\end{array}$

$\begin{array}{llllllll}\text { Pre- } \mathrm{Hb}<110 & 90 & 44 & 22 & 16 & 7 & 5 & 2\end{array}$

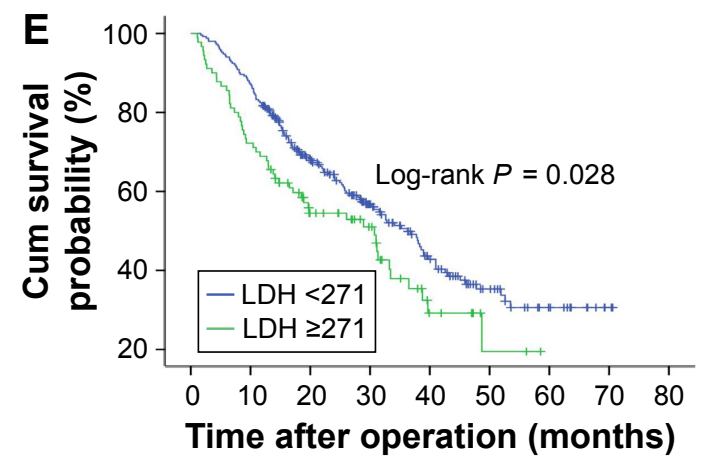

Number at risk

$\begin{array}{lllllllll}\text { Pre-LDH }<271 & 251 & 219 & 143 & 90 & 52 & 27 & 13 & 3\end{array}$

Pre-LDH $\geq 271 \quad 90 \quad 65 \quad 38 \quad 26 \quad 8 \quad 2$

Figure 2 Kaplan-Meier analysis of overall survival.

Notes: Unadjusted overall survival curves for independent factors by univariate analyses: depth of tumor invasion (A), age (B), resection margin (C), pre-Hb (D), and pre-LDH (E).

Abbreviations: pre-, preoperative; Hb, hemoglobin; LDH, lactic dehydrogenase; T, depth of tumor invasion; R0, positive resection margin; RI, negative resection margin.

\section{Discussion}

Surgical resection offers the only potentially curative option for BTC, ${ }^{5}$ while postoperative prognosis is unsatisfactory after extensive surgical resection. ${ }^{13-16}$ Moreover, the prognostic role of tumor markers has not yet been clearly elucidated in BTC patients undergoing radical surgery. Therefore, it is significant to identify prognostic factors that predict long-term survival of BTC patients who have undergone radical surgery. 
Table 4 Comparison between the high LDH and low LDH groups $(\mathrm{N}=34 \mathrm{I})$

\begin{tabular}{|c|c|c|c|}
\hline Variable & $\begin{array}{l}\text { LDH }<27 \text { I } \\
(\mathbf{N}=25 \text { I) } \\
\mathbf{N}(\%)\end{array}$ & $\begin{array}{l}\text { LDH } \geq 27 \text { I } \\
(\mathbf{N}=90) \\
\mathbf{N}(\%)\end{array}$ & $P$-value \\
\hline \multicolumn{4}{|l|}{ Age } \\
\hline$<65$ years & I 44 (57.4) & $5 \mathrm{I}(56.7)$ & 0.908 \\
\hline$\geq 65$ years & $107(42.6)$ & $39(43.3)$ & \\
\hline \multicolumn{4}{|l|}{ Sex } \\
\hline Male & $123(49.0)$ & $43(47.8)$ & 0.842 \\
\hline Female & $128(5 \mid .0)$ & $47(52.2)$ & \\
\hline \multicolumn{4}{|l|}{ Primary tumor site } \\
\hline Gallbladder & $99(39.8)$ & $28(31.5)$ & 0.154 \\
\hline Distal bile duct & $64(25.7)$ & $34(38.2)$ & \\
\hline Hilar & $58(23.3)$ & $17(19.1)$ & \\
\hline Intrahepatic & $28(11.2)$ & $10(11.2)$ & \\
\hline \multicolumn{4}{|l|}{ Depth of tumor invasion } \\
\hline TI & $20(8.4)$ & $3(3.4)$ & 0.064 \\
\hline $\mathrm{T} 2$ & $102(42.7)$ & $30(33.7)$ & \\
\hline T3 & $105(43.9)$ & $53(59.6)$ & \\
\hline $\mathrm{T} 4$ & $12(5.0)$ & $3(3.4)$ & \\
\hline \multicolumn{4}{|l|}{ Lymph node status } \\
\hline Negative & $170(70.2)$ & $53(58.9)$ & 0.050 \\
\hline Positive & $72(29.8)$ & $37(4 I . I)$ & \\
\hline \multicolumn{4}{|l|}{ Metastasis } \\
\hline Negative & $219(90.5)$ & $80(88.9)$ & 0.664 \\
\hline Positive & $23(9.5)$ & $10(11.1)$ & \\
\hline \multicolumn{4}{|l|}{ Seventh UICC TNM stage } \\
\hline 1 & $30(12.7)$ & II (I2.4) & 0.752 \\
\hline II & $104(44.1)$ & $34(38.2)$ & \\
\hline III & $69(29.2)$ & $31(34.8)$ & \\
\hline IV & $33(14.0)$ & $13(14.6)$ & \\
\hline \multicolumn{4}{|l|}{ Histologic differentiation } \\
\hline Well and moderately differentiated & II $3(48.5)$ & $38(43.7)$ & 0.442 \\
\hline Poorly differentiated & $120(51.5)$ & $49(56.3)$ & \\
\hline \multicolumn{4}{|l|}{ Lymphovascular invasion } \\
\hline Negative & $224(91.1)$ & $72(80.9)$ & 0.010 \\
\hline Positive & $22(8.9)$ & $17(19.1)$ & \\
\hline \multicolumn{4}{|l|}{ Perineural invasion } \\
\hline Negative & $135(54.9)$ & $37(41.6)$ & 0.031 \\
\hline Positive & III (45.I) & $52(58.4)$ & \\
\hline \multicolumn{4}{|l|}{ Resection margin } \\
\hline Negative & $194(78.9)$ & 64 (7I.9) & 0.182 \\
\hline Microscopic positive & $52(21.1)$ & $25(28.1)$ & \\
\hline Hemoglobin g/L, median (IQR) & $125.00(\mid 14.00-134.75)$ & $125(1 \mid 1.5-137.0)$ & 0.426 \\
\hline CEA ng/mL, median (IQR) & $3.10(2.06-5.22)$ & $3.80(2.30-5.50)$ & 0.503 \\
\hline CAI9-9 U/mL, median (IQR) & $68.15(19.35-336.90)$ & $132.50(55.60-707.40)$ & 0.192 \\
\hline
\end{tabular}

Note: Data in bold indicates $P<0.05$.

Abbreviations: LDH, lactic dehydrogenase; UICC, Union for International Cancer Control; TNM, tumor node metastasis; IQR, interquartile range; CEA, carcinoembryonic antigen; CAI9-9, carbohydrate antigen 19-9.

LDH has been reported to be a prognosticator in various types of tumors. ${ }^{22,24,26}$ Some studies suggest a prognostic role of LDH in patients with advanced BTC receiving certain chemotherapy. ${ }^{31,32}$ However, the prognosis of LDH in BTC patients undergoing radical surgery and in different tumor primary sites remains unclear. The present study revealed that high serum LDH level is significantly associated with worse survival in patients with operable BTC.
In our study, we discovered the prognostic roles of age, depth of tumor invasion, surgical margin status, hemoglobin, and serum LDH level for OS in multivariate analysis in the entire cohort of 341 patients surgically treated for BTC. Pradeep et $\mathrm{al}^{33}$ found that age was a significant predictor of survival by multivariate analysis in analyzing prognostic factors in 87 patients with GBC. Cubertafond et al ${ }^{14}$ reported in a survey of 724 patients surgically treated 
Table 5 Multivariate analysis of the patients with distal bile duct carcinoma $(\mathrm{N}=98)$

\begin{tabular}{|c|c|c|c|}
\hline \multirow[t]{2}{*}{ Variable } & \multicolumn{3}{|c|}{ Multivariate analysis } \\
\hline & HR & $95 \% \mathrm{Cl}$ & $P$-value \\
\hline Age $\geq 65$ years vs $<65$ years & 0.857 & $0.386-1.902$ & 0.704 \\
\hline Female vs male & 1.897 & $0.896-4.018$ & 0.094 \\
\hline \multicolumn{4}{|l|}{ Depth of tumor invasion } \\
\hline T3 vs T2 & 1.378 & $0.634-2.994$ & 0.418 \\
\hline T4 vs T2 & 1.142 & $0.111-11.715$ & 0.911 \\
\hline Lymph node status (positive vs negative) & 2.282 & $1.133-4.594$ & 0.021 \\
\hline Metastasis (positive vs negative) & 0.625 & $0.14 \mid-2.774$ & 0.537 \\
\hline \multicolumn{4}{|l|}{ Histologic differentiation } \\
\hline $\begin{array}{l}\text { Poorly vs well and moderately } \\
\text { differentiated }\end{array}$ & 1.135 & $0.529-2.436$ & 0.746 \\
\hline $\begin{array}{l}\text { Lymphovascular invasion (positive vs } \\
\text { negative) }\end{array}$ & 0.426 & $0.124-1.472$ & 0.177 \\
\hline Perineural invasion (positive vs negative) & 1.620 & $0.730-3.598$ & 0.236 \\
\hline Resection margin (positive vs negative) & 1.330 & $0.583-3.038$ & 0.498 \\
\hline Hemoglobin $(<110 \mathrm{~g} / \mathrm{L}$ vs $\geq 110 \mathrm{~g} / \mathrm{L})$ & 1.671 & $0.723-3.866$ & 0.230 \\
\hline CEA ( $\geq 4.7 \mathrm{ng} / \mathrm{mL}$ vs $<4.7 \mathrm{ng} / \mathrm{mL})$ & 1.069 & $0.478-2.392$ & 0.871 \\
\hline CAI9-9 ( $\geq 39 \mathrm{U} / \mathrm{mL}$ vs $<39 \mathrm{U} / \mathrm{mL})$ & 0.919 & $0.335-2.521$ & 0.870 \\
\hline $\mathrm{LDH}(\geq 27 \mathrm{I} \mathrm{U} / \mathrm{L}$ vs $<27$ I U/L) & 2.452 & $1.167-5.152$ & 0.018 \\
\hline
\end{tabular}

Note: Data in bold indicates $P<0.05$.

Abbreviations: CEA, carcinoembryonic antigen; CA19-9, carbohydrate antigen 19-9; LDH, lactic dehydrogenase.

for $\mathrm{GBC}$ that the long-term survival correlated with cancer stage: $>60$ months, $>22$ months, and 8 months for Tis, T1 to $\mathrm{T} 2$, and $\mathrm{T} 3$ to $\mathrm{T} 4$, respectively. $\mathrm{R} 0$ resection is reported to be prognosticator of long-term survival for patients with cholangiocarcinoma. ${ }^{10}$ These studies were consistent with our findings.

CA19-9 and CEA have been used as a complementary approach for the diagnosis of BTC in conjunction with radiology and histology/cytology, ${ }^{18-20}$ while the prognostic roles for better survival remain controversial. Lee et al ${ }^{35}$ suggested that CA19-9 but not CEA served as a predictor of better survival in patients with advanced cholangiocarcinoma on gemcitabine-based chemotherapy. Peixoto et al ${ }^{36}$ reported that CA19-9 and CEA were not significantly associated with OS in 106 patients with advanced BTC using univariate analysis. Our study showed a nonsignificant trend for better survival of patients with a low level of CA19-9 or CEA. The prognostic roles of CA19-9 and CEA for survival in BTC patients have to be further validated with a larger number of patients and prospective studies.

Moreover, we found that LDH is predictive of poor prognosis and clinical outcome in BTC patients undergoing radical surgery. In addition, Faloppi et $\mathrm{al}^{37}$ showed a possible prognostic role of pretreatment serum LDH levels in advanced BTC patients treated with first-line chemotherapy, confirming our hypothesis. In a Phase II study, Furuse et $\mathrm{a}^{31}$ revealed that elevated serum LDH level was associated with a significantly shorter survival in 85 patients with unresectable BTC receiving combination chemotherapy of uracil-tegafur and doxorubicin $(P=0.043)$. Moreover, these findings are in accordance with previously published analyses suggesting a relationship between LDH levels and a worse outcome in other tumor types. ${ }^{22,23,38}$ Furthermore, our study revealed that serum LDH can be a prognostic marker for DBD carcinoma. However, in terms of other primary tumors originating from the biliary tract, there were no statistically significant differences between LDH and OS in multivariate analysis in individual subgroups. The results indicated the possibility of different origins and biological behavior among these primary sites. Alternatively, this may be due to the limited number of patients in this study. Future studies may require greater numbers of patients to be considered statistically significant. The ABC- 02 trial demonstrated that the site of the primary tumor within the biliary tract did not affect survival. $^{7}$

Hypoxia-induced angiogenesis is probably the mechanism involved in high serum LDH levels and poor prognosis. ${ }^{22}$ Published studies demonstrated the association between LDH5 up-regulation and HIF (HIF $1 \alpha / \mathrm{HIF} 2 \alpha$ ) accumulation, which is linked with activating transcription of multiple genes including those encoding glycolytic enzymes and vascular endothelial growth factor. ${ }^{21,39}$ In addition, activated HIF pathway and angiogenic factor production are correlated with enhanced tumor aggressiveness. ${ }^{40,41}$

\section{Conclusion}

These data shed light on older age, depth of tumor invasion, positive surgical margin status, lower hemoglobin, and elevated serum LDH level as prognostic factors for poor survival in operable BTC patients. Moreover, serum LDH could be used as a cost-effective prognostic biomarker for DBD cancer. A limitation of this study is its retrospective nature, which means it lacks availability of some data, but it is reflective of the clinical spectrum of Chinese patients and is a relatively large study on this rare tumor type. In addition, this analysis is a single institutional series. These findings might provide new opportunities for prospective multi-institutional trials toward clinical applications of serum LDH in BTC patients undergoing radical surgery.

\section{Acknowledgments}

This study was partly supported by the following funding sources: the National Natural Science Foundation of China 
(No 81672896), the Priority Academic Program Development of Jiangsu Higher Education Institutions (JX10231802), Provincial Initiative Program for Excellency Disciplines, Jiangsu Province, China, CSCO-Merck tumor study funding (SCORE). No additional external funding was received for this study. The funders had no role in study design, data collection and analysis, decision to publish, or preparation of the manuscript.

\section{Disclosure}

The authors report no conflicts of interest in this work.

\section{References}

1. Siegel RL, Miller KD, Jemal A. Cancer statistics, 2017. CA Cancer J Clin. 2017;67(1):7-30.

2. Chen W, Zheng R, Baade PD, et al. Cancer statistics in China, 2015. CA Cancer J Clin. 2016;66(2):115-132.

3. Anderson CD, Pinson CW, Berlin J, Chari RS. Diagnosis and treatment of cholangiocarcinoma. Oncologist. 2004;9(1):43-57.

4. Howlader N, Noone A, Krapcho M, et al. SEER Cancer Statistics Review, 1975-2008. Bethesda: National Cancer Institute; 2015. Available from: http://seer.cancer.gov/csr/1975_2008/. Accessed April 9, 2018.

5. Khan SA, Davidson BR, Goldin RD, et al; British Society of Gastroenterology. Guidelines for the diagnosis and treatment of cholangiocarcinoma: an update. Gut. 2012;61(12):1657-1669.

6. Skipworth JR, Olde Damink SW, Imber C, Bridgewater J, Pereira SP, Malago M. Review article: surgical, neo-adjuvant and adjuvant management strategies in biliary tract cancer. Aliment Pharmacol Ther. 2011;34(9):1063-1078.

7. Valle J, Wasan H, Palmer DH, et al; ABC-02 Trial Investigators. Cisplatin plus gemcitabine versus gemcitabine for biliary tract cancer. N Engl J Med. 2010;362(14):1273-1281.

8. Andersen JB, Spee B, Blechacz BR, et al. Genomic and genetic characterization of cholangiocarcinoma identifies therapeutic targets for tyrosine kinase inhibitors. Gastroenterology. 2012;142(4):1021-1031. e1015.

9. Miller G, Socci ND, Dhall D, et al. Genome wide analysis and clinical correlation of chromosomal and transcriptional mutations in cancers of the biliary tract. J Exp Clin Cancer Res. 2009;28:62.

10. Murakami Y, Uemura K, Sudo T, et al. Prognostic factors after surgical resection for intrahepatic, hilar, and distal cholangiocarcinoma. Ann Surg Oncol. 2011;18(3):651-658.

11. Zhu GQ, Shi KQ, You J, et al. Systematic review with network metaanalysis: adjuvant therapy for resected biliary tract cancer. Aliment Pharmacol Ther. 2014;40(7):759-770.

12. Horgan AM, Amir E, Walter T, Knox JJ. Adjuvant therapy in the treatment of biliary tract cancer: a systematic review and meta-analysis. J Clin Oncol. 2012;30(16):1934-1940.

13. Sano T, Shimada K, Sakamoto Y, Yamamoto J, Yamasaki S, Kosuge T. One hundred two consecutive hepatobiliary resections for perihilar cholangiocarcinoma with zero mortality. Ann Surg. 2006;244(2):240-247.

14. Shaib YH, Davila JA, Henderson L, McGlynn KA, El-Serag HB. Endoscopic and surgical therapy for intrahepatic cholangiocarcinoma in the United States: a population-based study. J Clin Gastroenterol. 2007;41(10):911-917.

15. Nuzzo G, Giuliante F, Ardito F, et al. Intrahepatic cholangiocarcinoma: prognostic factors after liver resection. Updates Surg. 2010;62(1): $11-19$.

16. Ramacciato G, Nigri G, Bellagamba R, et al. Univariate and multivariate analysis of prognostic factors in the surgical treatment of hilar cholangiocarcinoma. Am Surg. 2010;76(11):1260-1268.
17. Primrose JN, Fox R, Palmer DH, et al. Adjuvant capecitabine for biliary tract cancer: the BILCAP randomized study. J Clin Oncol. 2017; 35(15_Suppl):4006.

18. Patel AH, Harnois DM, Klee GG, LaRusso NF, Gores GJ. The utility of CA 19-9 in the diagnoses of cholangiocarcinoma in patients without primary sclerosing cholangitis. Am J Gastroenterol. 2000;95(1): 204-207.

19. Wang YF, Feng FL, Zhao XH, et al. Combined detection tumor markers for diagnosis and prognosis of gallbladder cancer. World J Gastroenterol. 2014;20(14):4085-4092.

20. Grunnet M, Christensen IJ, Lassen U, et al. Decline in CA19-9 during chemotherapy predicts survival in four independent cohorts of patients with inoperable bile duct cancer. Eur J Cancer. 2015;51(11): 1381-1388.

21. Fantin VR, St-Pierre J, Leder P. Attenuation of LDH-A expression uncovers a link between glycolysis, mitochondrial physiology, and tumor maintenance. Cancer Cell. 2006;9(6):425-434.

22. Koukourakis MI, Giatromanolaki A, Sivridis E, et al; Tumour and Angiogenesis Research Group. Lactate dehydrogenase-5 (LDH-5) overexpression in non-small-cell lung cancer tissues is linked to tumour hypoxia, angiogenic factor production and poor prognosis. Br J Cancer. 2003;89(5):877-885.

23. Haas M, Heinemann V, Kullmann F, et al. Prognostic value of CA 19-9, CEA, CRP, LDH and bilirubin levels in locally advanced and metastatic pancreatic cancer: results from a multicenter, pooled analysis of patients receiving palliative chemotherapy. J Cancer Res Clin Oncol. 2013; 139(4):681-689.

24. Koukourakis MI, Giatromanolaki A, Sivridis E, Gatter KC, Harris AL, Tumour Angiogenesis Research Group. Lactate dehydrogenase 5 expression in operable colorectal cancer: strong association with survival and activated vascular endothelial growth factor pathway: a report of the Tumour Angiogenesis Research Group. J Clin Oncol. 2006;24(26):4301-4308.

25. Scartozzi M, Giampieri R, Maccaroni E, et al. Pre-treatment lactate dehydrogenase levels as predictor of efficacy of first-line bevacizumabbased therapy in metastatic colorectal cancer patients. Br J Cancer. 2012;106(5):799-804.

26. Petrelli F, Cabiddu M, Coinu A, et al. Prognostic role of lactate dehydrogenase in solid tumors: a systematic review and meta-analysis of 76 studies. Acta Oncol. 2015;54(7):961-970.

27. Bronsert P, Kohler I, Werner M, et al. Intestinal-type of differentiation predicts favourable overall survival: confirmatory clinicopathological analysis of 198 periampullary adenocarcinomas of pancreatic, biliary, ampullary and duodenal origin. BMC Cancer. 2013;13:428.

28. Nisman B, Lafair J, Heching N, et al. Evaluation of tissue polypeptide specific antigen, CYFRA 21-1, and carcinoembryonic antigen in nonsmall cell lung carcinoma: does the combined use of cytokeratin markers give any additional information? Cancer. 1998;82(10): $1850-1859$.

29. Yu H, Son GM, Joh YG. The clinical significance of preoperative serum levels of carbohydrate antigen 19-9 in colorectal cancer. J Korean Surg Soc. 2013;84(4):231-237.

30. Sobin LH, Gospodarowicz M, Wittekind C. TNM Classification of Malignant Tumors. Hoboken: Wiley-Blackwell; 2009.

31. Furuse J, Okusaka T, Ohkawa S, et al. A phase II study of uracil-tegafur plus doxorubicin and prognostic factors in patients with unresectable biliary tract cancer. Cancer Chemother Pharmacol. 2009;65(1): 113-120.

32. Saisho T, Okusaka T, Ueno H, Morizane C, Okada S. Prognostic factors in patients with advanced biliary tract cancer receiving chemotherapy. Hepatogastroenterology. 2004;52(66):1654-1658.

33. Pradeep R, Kaushik SP, Sikora SS, Bhattacharya BN, Pandey CM, Kapoor VK. Predictors of survival in patients with carcinoma of the gallbladder. Cancer. 1995;76(7):1145-1149. 
34. Cubertafond P, Gainant A, Cucchiaro G. Surgical treatment of 724 carcinomas of the gallbladder. Results of the French Surgical Association Survey. Ann Surg. 1994;219(3):275-280.

35. Lee BS, Lee SH, Son JH, et al. Prognostic value of CA 19-9 kinetics during gemcitabine-based chemotherapy in patients with advanced cholangiocarcinoma. J Gastroenterol Hepatol. 2016;31(2):493-500.

36. Peixoto RD, Renouf D, Lim H. A population based analysis of prognostic factors in advanced biliary tract cancer. $J$ Gastrointest Oncol. 2014;5(6):428-432.

37. Faloppi L, Del Prete M, Casadei Gardini A, et al. The correlation between LDH serum levels and clinical outcome in advanced biliary tract cancer patients treated with first line chemotherapy. Sci Rep. 2016;6:24136

38. Liu X, Meng QH, Ye Y, Hildebrandt MA, Gu J, Wu X. Prognostic significance of pretreatment serum levels of albumin, LDH and total bilirubin in patients with non-metastatic breast cancer. Carcinogenesis. 2015;36(2):243-248.
39. Koukourakis MI, Giatromanolaki A, Simopoulos C, Polychronidis A, Sivridis E. Lactate dehydrogenase 5 (LDH5) relates to up-regulated hypoxia inducible factor pathway and metastasis in colorectal cancer. Clin Exp Metastasis. 2005;22(1):25-30.

40. Blancher C, Moore JW, Talks KL, Houlbrook S, Harris AL. Relationship of hypoxia-inducible factor (HIF)-1 alpha and HIF-2 alpha expression to vascular endothelial growth factor induction and hypoxia survival in human breast cancer cell lines. Cancer Res. 2000;60(24):7106-7113.

41. Wang M, Zhao X, Zhu D, et al. HIF-1 $\alpha$ promoted vasculogenic mimicry formation in hepatocellular carcinoma through LOXL2 up-regulation in hypoxic tumor microenvironment. J Exp Clin Cancer Res. 2017; 36(1):60. 


\section{Supplementary materials}

Table SI Multivariate analysis of the patients with gallbladder carcinoma $(\mathrm{N}=127)$

\begin{tabular}{|c|c|c|c|}
\hline \multirow[t]{2}{*}{ Variable } & \multicolumn{3}{|c|}{ Multivariate analysis } \\
\hline & HR & $95 \% \mathrm{Cl}$ & $P$-value \\
\hline Age $\geq 65$ years vs $<65$ years & 2.389 & $1.356-4.209$ & 0.003 \\
\hline Female vs male & 0.589 & $0.338-1.025$ & 0.061 \\
\hline \multicolumn{4}{|l|}{ Depth of tumor invasion } \\
\hline T2 vs TI & 4.782 & $0.592-38.614$ & 0.142 \\
\hline T3 vs TI & 5.566 & $0.683-45.376$ & 0.109 \\
\hline T4 vs TI & 15.464 & $1.548-154.453$ & 0.020 \\
\hline Lymph node status (positive vs negative) & $1.28 \mathrm{I}$ & $0.688-2.383$ & 0.434 \\
\hline Metastasis (positive vs negative) & 1.601 & $0.752-3.406$ & 0.222 \\
\hline \multicolumn{4}{|l|}{ Histologic differentiation } \\
\hline Poorly vs well and moderately differentiated & 1.005 & $0.533-1.896$ & 0.987 \\
\hline Lymphovascular invasion (positive vs negative) & 1.642 & $0.746-3.612$ & 0.218 \\
\hline Perineural invasion (positive vs negative) & 1.336 & $0.749-2.384$ & 0.326 \\
\hline Resection margin (positive vs negative) & 1.715 & $0.887-3.314$ & 0.109 \\
\hline Hemoglobin $(<110 \mathrm{~g} / \mathrm{L}$ vs $\geq 110 \mathrm{~g} / \mathrm{L})$ & 1.651 & $0.772-3.533$ & 0.196 \\
\hline CEA $(\geq 4.7 \mathrm{ng} / \mathrm{mL}$ vs $<4.7 \mathrm{ng} / \mathrm{mL})$ & 1.462 & $0.767-2.786$ & 0.249 \\
\hline CAI9-9 ( $\geq 39 \mathrm{U} / \mathrm{mL}$ vs $<39 \mathrm{U} / \mathrm{mL})$ & 0.864 & $0.454-1.646$ & 0.657 \\
\hline $\mathrm{LDH}(\geq 27 \mid \mathrm{U} / \mathrm{L}$ vs $<27 \mid \mathrm{U} / \mathrm{L})$ & 1.735 & $0.864-3.483$ & 0.121 \\
\hline
\end{tabular}

Note: Data in bold indicates $P<0.05$.

Abbreviations: CEA, carcinoembryonic antigen; CA19-9, carbohydrate antigen 19-9; LDH, lactic dehydrogenase.

Table S2 Multivariate analysis of the patients with hilar carcinoma $(\mathrm{N}=75)$

\begin{tabular}{|c|c|c|c|}
\hline \multirow[t]{2}{*}{ Variable } & \multicolumn{3}{|c|}{ Multivariate analysis } \\
\hline & HR & $95 \% \mathrm{Cl}$ & $P$-value \\
\hline Age $\geq 65$ years vs $<65$ years & 0.697 & $0.268-1.815$ & 0.460 \\
\hline Female vs male & 1.528 & $0.591-3.948$ & 0.382 \\
\hline \multicolumn{4}{|l|}{ Depth of tumor invasion } \\
\hline $\mathrm{T} 2$ vs $\mathrm{TI}$ & 4.281 & $0.331-55.335$ & 0.265 \\
\hline T3 vs TI & 2.712 & $0.147-50.016$ & 0.502 \\
\hline T4 vs TI & 11.119 & $0.396-311.937$ & 0.157 \\
\hline Lymph node status (positive vs negative) & 0.618 & $0.140-2.731$ & 0.525 \\
\hline Metastasis (positive vs negative) & 0.140 & $0.015-1.289$ & 0.083 \\
\hline \multicolumn{4}{|l|}{ Histologic differentiation } \\
\hline Poorly vs well and moderately differentiated & 1.132 & $0.397-3.231$ & 0.817 \\
\hline Lymphovascular invasion (positive vs negative) & 1.493 & $0.348-6.409$ & 0.590 \\
\hline Perineural invasion (positive vs negative) & 0.300 & $0.115-0.782$ & 0.014 \\
\hline Resection margin (positive vs negative) & 1.676 & $0.662-4.239$ & 0.276 \\
\hline Hemoglobin $(<110 \mathrm{~g} / \mathrm{L}$ vs $\geq 110 \mathrm{~g} / \mathrm{L})$ & 1.373 & $0.262-7.196$ & 0.708 \\
\hline CEA $(\geq 4.7 \mathrm{ng} / \mathrm{mL}$ vs $<4.7 \mathrm{ng} / \mathrm{mL})$ & 1.889 & $0.697-5.119$ & 0.211 \\
\hline CAI9-9 ( $\geq 39 \mathrm{U} / \mathrm{mL}$ vs $<39 \mathrm{U} / \mathrm{mL})$ & 2.370 & $0.570-9.853$ & 0.235 \\
\hline $\mathrm{LDH}(\geq 27 \mid \mathrm{U} / \mathrm{L}$ vs $<27$ I U/L) & 2.200 & $0.561-8.627$ & 0.258 \\
\hline
\end{tabular}

Note: Data in bold indicates $P<0.05$.

Abbreviations: CEA, carcinoembryonic antigen; CA19-9, carbohydrate antigen 19-9; LDH, lactic dehydrogenase.

OncoTargets and Therapy

\section{Publish your work in this journal}

OncoTargets and Therapy is an international, peer-reviewed, open access journal focusing on the pathological basis of all cancers, potential targets for therapy and treatment protocols employed to improve the management of cancer patients. The journal also focuses on the impact of management programs and new therapeutic agents and protocols on

\section{Dovepress}

patient perspectives such as quality of life, adherence and satisfaction. The manuscript management system is completely online and includes a very quick and fair peer-review system, which is all easy to use. Visit http://www.dovepress.com/testimonials.php to read real quotes from published authors. 